\title{
Ionization Potentials within Semiempirical Antisymmetrized Product of Strictly Localized Geminals Approach
}

\author{
A. M. TOKMACHEV, A. L. TCHOUGRÉEFF, I. A. MISURKIN \\ L.Ya. Karpov Institute of Physical Chemistry, Vorontsovo pole 10, Moscow 103064, Russia
}

Received 14 August 2000; revised 16 February 2001; accepted 5 March 2001

\begin{abstract}
The APSLG-MINDO/3 method is evolved to reproduce the vertical ionization potentials for organic molecules. Two different schemes-one allowing for adjustment of the local electronic structure to the hole in each configuration and another one with a fixed local electronic structure-are developed and implemented. These methods are applied to a series of normal hydrocarbons. The possibility of localization of a hole in the polyethylene cation is discussed. Vertical ionization potentials are also obtained for a set of organic molecules with heteroatoms. Their values together with degeneracy of ionized states are compared with the data of photoelectron spectroscopy. (c) 2001 John Wiley \& Sons, Inc. Int J Quantum Chem 85: 109-117, 2001
\end{abstract}

Key words: ionization potentials; configuration interaction; semiempirical method; local electronic structure; geminals

\section{Introduction}

O alculations of vertical ionization potentials are usually performed on the basis of the HartreeFock approximation. Most workers in the field of photoelectron spectroscopy use the one-electron approximation and the Koopmans theorem [1] as primary tools to estimate the vertical ionization potentials. There also exist elaborated methods for calculating vertical ionization potentials that employ configuration interaction or perturbation ex-

Correspondence to: A. M. Tokmachev.

Contract grant sponsor: RFBR.

Contract grant number: 99-03-33176.

Contract grant sponsor: Haldor Topsøe A/S. pansions. Methods based on the Green function technique are also popular $[2,3]$. The success of the Hartree-Fock-based methods in explaining degeneracies of the energy eigenstates have led to a widespread point of view that the observed form of the photoelectron spectra confirms the orbital structure and the one-electron character of the molecular electronic wave functions. Thus it is interesting to explore methods for calculating ionization potentials that do not rely on the one-electron picture. It is also important to investigate the capacity of a method based on a local description of molecular electronic structure to describe the ionic states and to reproduce their symmetry properties, which ultimately are interpreted as delocalization. Some attempts in this direction were made in Refs. [4] and [5]. 
A local description of molecular electronic structure can be achieved by using the concept of geminals [6]. Our previous work [7, 8] was devoted to development of a semiempirical quantum chemical method that used the trial wave function of electrons taken as the antisymmetrized product of strictly localized geminals (APSLG) [9-11]. Starting from the MINDO/3 Hamiltonian, we modified its resonance parameters and obtained a computational scheme that turned out to be more efficient than the original MINDO/3 method based on the self-consistent field (SCF) approximation. The present work is devoted to an extension of the APSLG-MINDO/3 approximation [7, 8] toward calculation of ionization potentials.

Now we briefly recall the main features of the semiempirical APSLG-MINDO/3 approach [7, 8]. Within this approach, the basis set of atomic orbitals (AOs) is replaced by a set of hybrid orbitals (HOs). The transition to the local description is carried out by a unitary transformation (with optimized parameters of the latter) on each "heavy" (nonhydrogen) atom. Each $\mathrm{HO}$ is assigned to a chemical bond or an electron lone pair. Therefore, each $(m \mathrm{th})$ chemical bond is expanded through two HOs: $\left|r_{m}\right\rangle$ and $\left|l_{m}\right\rangle$ (right and left). An APSLG-type wave function is constructed as an antisymmetrized product of the geminals-two-electron functions. In the second quantization language it can be written as

$$
|\Phi\rangle=\prod_{m} g_{m}^{+}|0\rangle .
$$

Each geminal can be presented as a sum of three singlet two-electron configurations in the basis of four spin-orbitals assigned to this chemical bond,

$$
g_{m}^{+}=u_{m} r_{m \alpha}^{+} r_{m \beta}^{+}+v_{m} l_{m \alpha}^{+} l_{m \beta}^{+}+w_{m}\left(r_{m \alpha}^{+} l_{m \beta}^{+}+l_{m \alpha}^{+} r_{m \beta}^{+}\right)
$$

with variable amplitudes $u_{m}, v_{m}$, and $w_{m}$. The first and the second terms correspond to the ionic configurations with two electrons on the same (the right or the left, respectively) end of the chemical bond. The third term represents the covalent contribution to the geminal, i.e., it is analogous to the Heitler-London wave function of the dihydrogen molecule [12]. In the case of electron lone pairs, only one of the ionic terms (the right one, for the sake of definiteness) does not vanish. The normalization condition is imposed on the amplitudes of the geminal expansion (2):

$$
\left\langle 0\left|g_{m} g_{m}^{+}\right| 0\right\rangle=u_{m}^{2}+v_{m}^{2}+2 w_{m}^{2}=1 .
$$

The MINDO/3-type Hamiltonian is transformed to the $\mathrm{HO}$ basis. In this case it also can be represented as a sum of intraatom (one-center) and interatom (two-center) contributions

$$
\mathbf{H}=\sum_{A} \mathbf{H}_{A}+\frac{1}{2} \sum_{A \neq B} \mathbf{H}_{A B},
$$

where the intraatom contribution is

$$
\begin{aligned}
& \mathbf{H}_{A}= \sum_{t_{m} \in A}\left(U_{m}^{t}-\sum_{B \neq A} \gamma_{A B} Z_{B}\right) \sum_{\sigma} t_{m \sigma}^{+} t_{m \sigma} \\
&-\sum_{t_{m_{1}} t_{m_{2}} \in A} \beta_{t_{m_{1}} t_{m_{2}}^{\prime}}^{A} \sum_{\sigma}\left(t_{m_{1} \sigma}^{+} t_{m_{2} \sigma}^{\prime}+\text { h.c. }\right) \\
&+ \frac{1}{2} \sum_{t_{m_{1}} t_{m_{2}}^{\prime}}\left(t_{m_{1}} t_{m_{2}}^{\prime} \mid t_{m_{3}}^{\prime \prime} t_{m_{4}}^{\prime \prime \prime}\right)^{A} \\
& t_{m_{3}}^{\prime \prime} t_{m_{4}}^{\prime \prime \prime} \in A \\
& \times \sum_{\sigma \tau} t_{m_{1} \sigma}^{+} t_{m_{3} \tau}^{\prime+} t_{m_{4} \tau}^{\prime \prime \prime} t_{m_{2} \sigma}^{\prime}
\end{aligned}
$$

whereas the interatom contribution is

$$
\begin{aligned}
\mathbf{H}_{A B}=-\sum_{\substack{t_{m_{1}} \in A \\
t_{m_{2}}^{\prime} \in B}} \beta_{m_{1} m_{2}}^{A B} \sum_{\sigma}\left(t_{m_{1} \sigma}^{+} t_{m_{2} \sigma}^{\prime}+\text { h.c. }\right) \\
+\gamma_{A B} \sum_{\substack{t_{m_{1}} \in A \\
t_{m_{2}}^{\prime} \in B}} \sum_{\sigma \tau} t_{m_{1} \sigma}^{+} t_{m_{2} \tau}^{+} t_{m_{2} \tau}^{\prime} t_{m_{1} \sigma},
\end{aligned}
$$

where h.c. stands for hermitian conjugation and $\left|t_{m}\right\rangle$ denotes one of the two HOs (right or left) that belong to the $m$ th geminal. The optimal HOs and the amplitudes $\left(u_{m}, v_{m}\right.$, and $\left.w_{m}\right)$ are determined variationally. The results of our calculations have shown that the accuracy of the APSLG-MINDO/3 method is comparable and somewhat better than that of the SCF-MINDO/3 method when it is applied to the heats of formation and equilibrium geometries of organic molecules. The dependence of the calculation time on the size of the system (i.e., the number of basis functions) is linear [8]. Also the APSLG (but not SCF) approach ensures a correct asymptotic of the wave function under bond cleavage.

\section{Theory}

Now we consider a method for deriving vertical ionization potentials for the APSLG-type wave function. In the framework of the APSLG method, it is very natural to construct $(N-1)$-electron states by extracting an electron from one of the geminals and conserving the geminals structures for other bonds and/or lone pairs, similarly to the way the ionized states are constructed for the SCF method [1]. At the same time, the states obtained by extracting an electron from a geminal with other geminals unchanged 
are not even approximately the eigenstates of the ion. They do not permit the form of the photoelectron spectra to be explained. To obtain the correct eigenstates of the ion, we take into account the interaction between these $(N-1)$-electron states. The way to construct these states is the key point of this article.

When one electron is extracted from the geminal, the remaining electron can occupy any of the four spin-orbitals in the case of a geminal that represents a usual chemical bond or one of two spin-orbitals in the case of a geminal that represents an electron lone pair. If we assume these spin-orbitals to be $\left|r_{m \sigma}\right\rangle$ and $\left|l_{m \sigma}\right\rangle$, we obtain configurations that strongly interact due to the large intrabond resonance terms. In this context, it is more natural to use so-called bond orbitals (BOs), which constitute an alternative one-electron basis set. The BOs related to a given geminal (bond) are expanded in terms of the corresponding HOs:

$$
\begin{aligned}
& b_{m \sigma}=x_{m} l_{m \sigma}+y_{m} r_{m \sigma}, \\
& a_{m \sigma}=-y_{m} l_{m \sigma}+x_{m} r_{m \sigma} .
\end{aligned}
$$

These orbitals are also normalized,

$$
x_{m}^{2}+y_{m}^{2}=1,
$$

and orthogonal,

$$
\left\langle 0\left|a_{m \sigma} b_{m \sigma}^{+}\right| 0\right\rangle=\left\langle 0\left|b_{m \sigma} a_{m \sigma}^{+}\right| 0\right\rangle=0 .
$$

The coefficients $x_{m}$ and $y_{m}$ are determined on the basis of the $L$ orthogonality condition (i.e., the states generated by the action of the BO-related fermion operators on the ground state of the molecule in the APSLG approximation are orthogonal):

$$
\left\langle 0\left|g_{m} a_{m \sigma} b_{m \sigma}^{+} g_{m}^{+}\right| 0\right\rangle=\left\langle 0\left|g_{m} b_{m \sigma} a_{m \sigma}^{+} g_{m}^{+}\right| 0\right\rangle=0 .
$$

If we denote the coefficients as $x_{m}=\cos \varphi_{m}$ and $y_{m}=\sin \varphi_{m}$, then the angle $\varphi_{m}$ is determined by the expression

$$
\varphi_{m}=\frac{1}{2} \arctan \left(\frac{2 w_{m}}{v_{m}-u_{m}}\right) .
$$

Note that the orbitals defined by Eq. (7) are, respectively, usual bonding and antibonding orbitals for the $m$ th bond. The reciprocal transition to the atomic HOs is given by

$$
\begin{aligned}
& r_{m \sigma}=x_{m} a_{m \sigma}+y_{m} b_{m \sigma}, \\
& l_{m \sigma}=x_{m} b_{m \sigma}-y_{m} a_{m \sigma} .
\end{aligned}
$$

The geminal of the $m$ th bond can be rewritten in terms of bonding and antibonding BOs,

$$
g_{m}^{+}=U_{m} b_{m \alpha}^{+} b_{m \beta}^{+}+V_{m} a_{m \alpha}^{+} a_{m \beta}^{+},
$$

where the new coefficients of the geminal expansion comply with the following normalization condi- tions for the geminals:

$$
U_{m}^{2}+V_{m}^{2}=1 .
$$

Note that the representation of the geminal in Eq. (13) is simply its natural form. By substituting Eq. (7) into Eq. (13) and comparing the result with Eq. (2), we obtain that the coefficients in Eq. (13) are

$$
\begin{aligned}
& U_{m}=\frac{1}{2}\left(u_{m}+v_{m}+\sqrt{\left(u_{m}-v_{m}\right)^{2}+4 w_{m}^{2}}\right), \\
& V_{m}=\frac{1}{2}\left(u_{m}+v_{m}-\sqrt{\left(u_{m}-v_{m}\right)^{2}+4 w_{m}^{2}}\right) .
\end{aligned}
$$

The reverse relationship between the amplitudes of the geminal in terms of the atomic $\mathrm{HOs}$ and $\mathrm{BOs}$ is unique and can be written as

$$
\begin{aligned}
u_{m} & =U_{m} y_{m}^{2}+V_{m} x_{m}^{2}, \\
v_{m} & =U_{m} x_{m}^{2}+V_{m} y_{m}^{2} \\
w_{m} & =\left(U_{m}-V_{m}\right) x_{m} y_{m} .
\end{aligned}
$$

Both basis sets (BOs and HOs) will be used hereafter. It is necessary to mention that in the case of electron lone pairs, we can construct only one $\mathrm{BO}$ that coincides with the $\mathrm{HO}$.

Now we consider a method for constructing the ionized states. After one electron is extracted from a geminal, the remaining electron may occupy either the bonding or antibonding $\mathrm{BO}$. All other bonds are represented by their ground state geminals. Thus generated $(N-1)$-electron states are

$$
\left|\Phi_{c_{m \sigma}}^{+}\right\rangle=\left(\prod_{k \neq m} g_{k}^{+}\right) c_{m \sigma}^{+}|0\rangle,
$$

where the fermion creation operator $c_{m \sigma}^{+}$stands for either of the fermion operators $\left(b_{m \sigma}^{+}\right.$or $\left.a_{m \sigma}^{+}\right)$that creates electrons with the spin projection $\sigma$. The Hamiltonian Eq. (4) does not contain contributions that allow the states in Eq. (17) with different spin projections to interact. Therefore, the eigenstates of the ion can be presented by a linear combination of the basis functions with the same spin projection:

$$
\left|\Psi_{n \sigma}^{+}\right\rangle=\sum_{c_{m}} \chi_{c_{m}}^{n}\left|\Phi_{c_{m \sigma}}^{+}\right\rangle
$$

The coefficients $\chi_{c_{m}}^{n}$ are determined by solving the eigenvalue problem

$$
\mathbf{H}\left|\Psi_{n \sigma}^{+}\right\rangle=E_{n}^{+}\left|\Psi_{n \sigma}^{+}\right\rangle .
$$

The choice of basis ion states in the form Eq. (17) leads to a simple matrix form of the eigenvalue problem with the unity matrix as a metric. If the $(N-1)$-electron states were obtained by the action of some basis electron annihilation operators 
on the ground state of the molecule, the eigenvalue problem would have the density matrix as a metric, which immediately leads to the problem of occasionally small occupation numbers. The vertical ionization potentials must be obtained by subtracting the energy of the ground state of the neutral molecule $E_{0}$ taken in the APSLG approximation from the eigenenergies $E_{n}^{+}$of the ion. If we replace the Hamiltonian in Eq. (19) by the operator $\mathbf{H}-E_{0} \mathbf{I}$, the corresponding eigenvalue problem gives the eigenstates of the ion and the vertical ionization potentials as its eigenvalues. An analogous method was considered in Ref. [4] for the localized wave function of the Hartree-Fock method. Moreover, assuming the transferability of the matrix elements of the configuration interaction matrix, the simple scheme of evaluating the ionization potentials similar to the Hückel method, but with atoms replaced by bonds, was constructed.

Now we turn to an analysis of the matrix elements of $\mathbf{H}-E_{0} \mathbf{I}$. First we consider the diagonal elements of the operator $\mathbf{H}-E_{0} \mathbf{I}$. Let us denote

$$
\eta_{m}^{t c}=\left\langle 0\left|t_{m \sigma} c_{m \sigma}^{+}\right| 0\right\rangle .
$$

This quantity is the coefficient of the $\mathrm{BO}\left|c_{m}\right\rangle$ in the expansion for the $\mathrm{HO}\left|t_{m}\right\rangle$ [see Eq. (12)]. Also, if $\left|t_{m}\right\rangle$ is one of the two HOs $\left|r_{m}\right\rangle$ or $\left|l_{m}\right\rangle$, then $T_{m}$ stands for the atom on which the orbital $\left|t_{m}\right\rangle$ is centered ( $R_{m}$ or $L_{m}$, respectively). The matrix elements of the one-electron density matrix are $[7,8]$

$$
\begin{aligned}
& P_{m}^{t t^{\prime}}=\left\langle 0\left|g_{m} t_{m \sigma}^{+} t_{m \sigma}^{\prime} g_{m}^{+}\right| 0\right\rangle, \quad P_{m}^{r r}=u_{m}^{2}+w_{m}^{2}, \\
& P_{m}^{l l}=v_{m}^{2}+w_{m}^{2}, \quad P_{m}^{r l}=P_{m}^{l r}=\left(u_{m}+v_{m}\right) w_{m} .
\end{aligned}
$$

The matrix elements of the two-electron density matrix are $[7,8]$

$$
\begin{aligned}
\Gamma_{m}^{t t^{\prime}} & =\left\langle 0\left|g_{m} t_{m \sigma}^{+} t_{m-\sigma}^{++} t_{m-\sigma}^{\prime} t_{m \sigma} g_{m}^{+}\right| 0\right\rangle, \\
\Gamma_{m}^{r r} & =u_{m}^{2}, \quad \Gamma_{m}^{l l}=v_{m}^{2}, \quad \Gamma_{m}^{r l}=\Gamma_{m}^{l r}=w_{m}^{2} .
\end{aligned}
$$

Using these notations, we obtain

$$
\begin{aligned}
H_{c_{m} c_{m}}= & \left\langle 0\left|\left(\prod_{k^{\prime} \neq m} g_{k^{\prime}}\right) c_{m \sigma}\left(\mathbf{H}-E_{0} \mathbf{I}\right) c_{m \sigma}^{+}\left(\prod_{k \neq m} g_{k}^{+}\right)\right| 0\right\rangle \\
= & \sum_{t \in\{r, l\}}\left\{\left(U_{m}^{t}-\sum_{B \neq T_{m}} \gamma_{T_{m} B} Z_{B}\right)\left[\left(\eta_{m}^{t c}\right)^{2}-2 P_{m}^{t t}\right]\right. \\
& \left.\quad-\left(t_{m} t_{m} \mid t_{m} t_{m}\right)^{T_{m}} \Gamma_{m}^{t t}\right\} \\
& -2 \beta_{r_{m} l_{m}}^{R_{m} L_{m}}\left(\eta_{m}^{r c} \eta_{m}^{l c}-2 P_{m}^{r l}\right)-2 \gamma_{R_{m} L_{m}} \Gamma_{m}^{r l} \\
& +\sum_{t \in\{r, l\}}\left[\left(\eta_{m}^{t c}\right)^{2}-2 P_{m}^{t t}\right]
\end{aligned}
$$

$$
\begin{aligned}
& \times \sum_{\substack{t_{q}^{\prime} \in T_{m} \\
q \neq m}}\left[2\left(t_{m} t_{m} \mid t_{q}^{\prime} t_{q}^{\prime}\right)^{T_{m}}\right. \\
+ & \left.2 \sum_{t \in\{r, l\}}\left[\left(\eta_{m}^{t c}\right)^{2}-2 t_{q}^{\prime} \mid t_{m} t_{q}^{\prime}\right)^{T_{m}}\right] \sum_{B \neq T_{m}} \gamma_{B T_{m}} \sum_{\substack{t_{q}^{\prime} \in B \\
q \neq m \\
q \neq m}} P_{q}^{t^{\prime} t^{\prime}} .
\end{aligned}
$$

The off-diagonal matrix elements also can be divided in two classes: (1) those between the ionized states with an electron extracted from one geminal but from the different BOs assigned to the latter and (2) those between two ionized states with an electron extracted from two different geminals. Let us consider the first case. Let us set

$$
\bar{c}_{m \sigma}= \begin{cases}a_{m \sigma}, & \text { if } c_{m \sigma}=b_{m \sigma}, \\ b_{m \sigma}, & \text { if } c_{m \sigma}=a_{m \sigma} .\end{cases}
$$

Then

$$
\begin{aligned}
& H_{c_{m} \bar{c}_{m}}=\left\langle 0\left|\left(\prod_{k^{\prime} \neq m} g_{k^{\prime}}\right) c_{m \sigma}\left(\mathbf{H}-E_{0} \mathbf{I}\right) \bar{c}_{m \sigma}^{+}\left(\prod_{k \neq m} g_{k}^{+}\right)\right| 0\right\rangle \\
& =\sum_{t \in\{r, l\}}\left(U_{m}^{t}-\sum_{B \neq T_{m}} \gamma_{T_{m} B} Z_{B}\right) \eta_{m}^{t c} \eta_{m}^{t \bar{c}} \\
& -\beta_{r_{m} l_{m}}^{R_{m} L_{m}}\left(\eta_{m}^{r c} \eta_{m}^{l \bar{c}}+\eta_{m}^{r \bar{c}} \eta_{m}^{l c}\right) \\
& +\sum_{t \in\{r, l\}} \eta_{m}^{t c} \eta_{m}^{t \bar{c}} \sum_{t_{q}^{\prime} \in T_{m}}\left[2\left(t_{m} t_{m} \mid t_{q}^{\prime} t_{q}^{\prime}\right)^{T_{m}}\right. \\
& \left.-\left(t_{m} t_{q}^{\prime} \mid t_{m} t_{q}^{\prime}\right)^{T_{m}}\right] P_{q}^{t^{\prime} t^{\prime}} \\
& +2 \sum_{t \in\{r, l\}} \eta_{m}^{t c} \eta_{m}^{t \bar{c}} \sum_{B \neq T_{m}} \gamma_{B T_{m}} \sum_{\substack{t_{q}^{\prime} \in B \\
q \neq m}} P_{q}^{t^{\prime} t^{\prime}} .
\end{aligned}
$$

Let us denote

$$
C_{m c}= \begin{cases}U_{m}, & \text { if } c_{m \sigma}=b_{m \sigma}, \\ V_{m}, & \text { if } c_{m \sigma}=a_{m \sigma} .\end{cases}
$$

The off-diagonal matrix element of the Hamiltonian between the ionized states obtained by the electron extraction from the different geminals can be written as

$$
\begin{aligned}
H_{c_{m} c_{n}^{\prime}}=\left\langle 0\left|\left(\prod_{k^{\prime} \neq m} g_{k^{\prime}}\right) c_{m \sigma}\left(\mathbf{H}-E_{0} \mathbf{I}\right) c_{n \sigma}^{+}\left(\prod_{k \neq n} g_{k}^{+}\right)\right| 0\right\rangle \\
=\sum_{t t^{\prime} \in\{r, l\}} C_{m c} C_{n c^{\prime}} \eta_{m}^{t c} \eta_{n}^{t^{\prime} c^{\prime}} \\
\times\left\{\beta_{t_{m} t_{n}^{\prime}}^{T_{m} T_{n}^{\prime}}\right. \\
\quad-\delta_{T_{m} T_{n}^{\prime}} \sum_{\substack{t_{q}^{\prime \prime} \in T_{m} \\
q \neq m, n}} P_{q}^{t^{\prime \prime} t^{\prime \prime}}\left[2\left(t_{q}^{\prime \prime} t_{q}^{\prime \prime} \mid t_{m} t_{n}^{\prime}\right)^{T_{m}}\right. \\
\left.\left.-\left(t_{q}^{\prime \prime} t_{m} \mid t_{q}^{\prime \prime} t_{n}^{\prime}\right)^{T_{m}}\right]\right\}
\end{aligned}
$$




$$
\begin{aligned}
& -\sum_{t t^{\prime} \in\{r, l\}} \delta_{T_{m} T_{n}^{\prime}} \eta_{m}^{t c} \eta_{n}^{t^{\prime} c^{\prime}}\left(t_{m} t_{m} \mid t_{m} t_{n}^{\prime}\right)^{T_{m}} \\
& \quad \times C_{n c^{\prime}}\left[\left(\eta_{m}^{t a}\right)^{2} C_{m a}+\left(\eta_{m}^{t b}\right)^{2} C_{m b}\right] \\
& -\sum_{t t^{\prime} \in\{r, l\}} \delta_{T_{m} T_{n}^{\prime}} \eta_{m}^{t c} \eta_{n}^{t^{\prime} c^{\prime}}\left(t_{n}^{\prime} t_{n}^{\prime} \mid t_{n}^{\prime} t_{m}\right)^{T_{m}} \\
& \quad \times C_{m c}\left[\left(\eta_{n}^{t^{\prime} a}\right)^{2} C_{n a}+\left(\eta_{n}^{t^{\prime} b}\right)^{2} C_{n b}\right] .
\end{aligned}
$$

In the case of lone pairs, these formulae remain true, but we must consider only the states of type $\left|\Phi_{c_{m \sigma}}^{+}\right\rangle$ and the contributions from the "right-end" atom. Due to the spin projection conservation rules, the overall dimension of the matrix to be diagonalized is $2 M+N$, where $M$ is the number of chemical bonds and $N$ is the number of electron lone pairs in the molecule.

Another (more sophisticated) possibility for obtaining the vertical ionization potentials and the corresponding eigenstates is to allow the nonionized geminals to relax to accommodate the hole. Such a procedure must be performed separately for each many-electron basis state. The whole procedure leads to basis states analogous to Eq. (17), but with the geminals not taken from the ground state of the neutral molecule, but obtained via an optimization procedure for $(M+N-1)$ geminals in the system with an electron extracted from one of geminals of the molecule under consideration. In a molecule with a hole, the effective Hamiltonians for each geminal differ from those in the neutral molecule. Thus the optimal geminals for the ionized molecule differ from those in the neutral molecule as well.

The above-mentioned adjustment of the geminals to the presence of the hole is nothing but polarization of these geminals in the Coulomb field induced by the hole. Polarization of the SCF localized wave function by the hole was investigated in the Ref. [5] in second order perturbation theory. Let us consider a method that takes into account the polarization of the geminals in the presence of a hole. To this end, we construct the effective Hamiltonians for each of the geminals in the field of other geminals, including the field where the hole is residing $\left(H_{k c_{m \tau}}^{\text {eff }}\right)$. The explicit form of the effective Hamiltonians is presented in Appendix. The polarized geminals can be obtained by solving the eigenvalue problem

$$
H_{k c_{m \tau}}^{\mathrm{eff}} \tilde{g}_{k c_{m \tau}}=\tilde{\epsilon}_{k c_{m \tau}} \tilde{g}_{k c_{m \tau}}
$$

where $\tilde{g}_{k c_{m \tau}}$ is the $k$ th geminal polarized by the hole on the $m$ th geminal with the remaining electron residing on the bond spin-orbital $\left|c_{m \tau}\right\rangle$. Expressions for the matrix elements of the operator $\mathbf{H}-E_{0} \mathbf{I}$ in the basis of the ionized basis states with the adjusted (polarized) geminals are very cumbersome and will not be presented here. The main difference between the off-diagonal matrix elements of the two methods can be termed multiplication by factors similar to the product of the overlap integrals between the geminals $\tilde{g}_{k c_{m \tau}}$ with different values of $k$. The diagonal matrix elements are shifted due to changes in the contributions to the Hamiltonian from the nonionized geminals caused by their polarization.

\section{Results and Discussion}

We have implemented the two previously mentioned computational procedures that determine the vertical ionization potentials. The APSLGMINDO/3 approximation [7, 8] was employed to calculate the ground states of these molecules and to parameterize the Hamiltonian matrices for the ionized states. The specific structure of the APSLG approximation restricts these schemes only to the molecules that can be represented by structures with well defined separate chemical bonds. First we compare both procedures with the results of the SCF-MINDO/3 method for a series of normal hydrocarbons. The higher members of this series simulate the polyethylene chain. The first question in this context concerns whether the structure of the hole in the higher hydrocarbons is localized on several chain segments or delocalized over the whole chain. The one-electron approximation results in a plane-wave-like structure for the wave function of the lowest ionized state (ground state of the cation), i.e., the coefficient of the $k$ th orbital on the $n$th atom in the hydrocarbon molecule $\mathrm{C}_{N} \mathrm{H}_{2 N+2}$ is

$$
c_{n k}=\sqrt{\frac{2}{N+1}} \sin \frac{\pi k n}{N+1} .
$$

(In this model, one orbital per methyl or methylene group in the chain is assumed. These orbitals correspond to the Wannier states derived from the upper filled band.) At the same time, the variation of the lengths of chemical bonds in this simplest model yields the localized ionized state (or polaronic state) [13]. In the context of our model, the following question is worth study: Does the method operating with local entities like geminals yield a localized or delocalized description of a hole? Such a localized state would corresponded to a polaronic state with only noninertial (electronic) polarization taken into account. Another question to be studied 
for a series of hydrocarbons is the dependence of the vertical ionization potential on the hydrocarbon chain length.

The dependencies obtained by the SCFMINDO/3 method, the APSLG-MINDO/3 method without polarization of the geminals and the APSLG-MINDO/3 method that takes into account the polarization of geminals in the presence of the hole are given in Table I. The geometry structures of the neutral molecules in all these cases were obtained by minimizing the energy of the molecule in its ground state by the respective method (SCF-MINDO/3 or APSLG-MINDO/3). Figure 1 represents all three dependencies compared with the data of photoelectron spectroscopy. The analysis of these data reveals that both the APSLG-based methods described in the previous section give values of vertical ionization potentials that are lower than the SCF method does. As we expected, the vertical ionization potentials obtained by the APSLG-MINDO/3 method with the polarized geminals are always lower than those obtained by the APSLG-MINDO/3 method with the fixed geminals. This is due to a larger number of degrees of freedom

TABLE I

The first vertical ionization potentials (in $\mathrm{eV}$ ) in a series of hydrocarbons from $\mathrm{CH}_{4}$ to $\mathrm{C}_{20} \mathrm{H}_{42}$ obtained by SCF-MINDO/3 and by both APLSG-based procedures.

\begin{tabular}{rrrr}
\hline$N$ & & $\begin{array}{c}\text { Fixed } \\
\text { Geminals }\end{array}$ & $\begin{array}{r}\text { Polarized } \\
\text { Geminals }\end{array}$ \\
\hline 1 & SCF & 13.306 & 13.079 \\
2 & 13.289 & 11.657 & 11.212 \\
3 & 11.758 & 11.130 & 10.572 \\
4 & 11.291 & 10.862 & 10.266 \\
5 & 11.044 & 10.600 & 10.034 \\
6 & 10.916 & 10.383 & 9.789 \\
7 & 10.722 & 10.226 & 9.612 \\
8 & 10.606 & 10.107 & 9.476 \\
9 & 10.514 & 10.015 & 9.371 \\
10 & 10.424 & 9.942 & 9.288 \\
11 & 10.368 & 9.885 & 9.222 \\
12 & 10.328 & 9.838 & 9.169 \\
13 & 10.279 & 9.800 & 9.125 \\
14 & 10.232 & 9.768 & 9.089 \\
15 & 10.220 & 9.742 & 9.059 \\
16 & 10.192 & 9.720 & 9.034 \\
17 & 10.172 & 9.701 & 9.012 \\
18 & 10.151 & 9.685 & 8.994 \\
19 & 10.136 & 9.671 & 8.978 \\
20 & 10.108 & 9.658 & 8.964 \\
\hline & 10.105 & & \\
\hline & & & \\
\hline
\end{tabular}

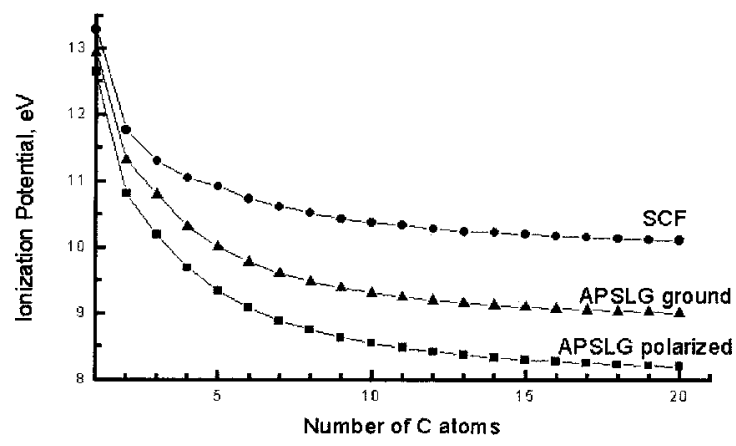

FIGURE 1. Vertical ionization potentials in a series of hydrocarbons.

in the case of the polarized geminals. The numerical values of the vertical ionization potential obtained by the APSLG-MINDO/3-based method with fixed geminals are in a good agreement with the experimental data. It is necessary to mention that the experimental data on the ionization potentials are very divergent (see, for example, Refs. [14-16] for methane and Refs. [14, 15, 17], and [18] for ethane and propane). At the same time, the values of the vertical ionization potential obtained by the APSLG-MINDO/3-based method with polarized geminals are noticeably (by more than $0.5 \mathrm{eV}$ ) lower. So, the photoionization experiment for the $\mathrm{C}_{11} \mathrm{H}_{24}$ molecule [19] gives a value for the first adiabatic ionization potential equal to $9.6 \mathrm{eV}$. The APSLG-MINDO/3-based method with the polarized geminals gives a value of the vertical ionization potential that is $0.4 \mathrm{eV}$ lower than the experimental adiabatic value. This can be readily understood when we compare the ways to obtain the ground state energy for neutral molecules and their positive ions. The wave function of the ion with fixed geminals has approximately the same level of correlation as the wave function of the neutral molecule. At the same time, in the case of the APSLG-based method with polarized geminals, this balance is broken.

It is interesting to reproduce the positions of the peaks in the photoelectron spectrum. For example, in the case of methane, the experimental data give the second peak in the photoelectron spectrum at about $23 \mathrm{eV}$ [20], the SCF-MINDO/3 method gives $27 \mathrm{eV}$ for this quantity, and the APSLG-MINDO/3 method with the fixed geminals gives $26.3 \mathrm{eV}$ (the polarization of geminals lowers this value by $0.1 \mathrm{eV}$ only). Thus, both semiempirical quantum chemical methods push this value essentially higher than experiment. In the case of highly symmetric mole- 
cules, their photoionization yields degenerate ionized states. For example, in the case of methane, a triply degenerate peak near $13 \mathrm{eV}$ is observed. In the case of ethane, a doubly degenerate peak near $11.5 \mathrm{eV}$ is observed. These experimental data are explained theoretically on the basis of orbital theories (see, for example, [21, 22]). The origin of these peaks is ascribed to extraction of an electron from the higher triply degenerate molecular orbital in the case of methane (the $T_{2}$ representation of the $T_{d}$ point group, which is the symmetry group of the Fock operator for the methane molecule) and from the doubly degenerate molecular orbital in the case of ethane. This fact for years served as an argument in favor of the orbital picture of the electronic structure of a molecule, although, in fact, it is a consequence of the SCF approximation. Therefore, it was interesting to consider the capacity of the APSLG approximation to correctly reproduce the form of the photoelectron spectra. In the framework of the preceding scheme, we studied the degeneracy of the ionized states for methane and ethane molecules. It turns out that the experimental structure of the spectrum is reproduced by our calculations. Of course, the spectrum is totally controlled by the symmetry of configuration interaction matrix. In the case of methane, the Hamiltonian matrix consists of four equal diagonal $2 \times 2$ blocks. The off-diagonal $2 \times 2$ blocks are also equal. This leads to a spectrum with two triply degenerate and two nondegenerate eigenvalues.

Another question to be studied is localization of the hole in polyethylene (or, in our case, in higher hydrocarbons). The eigenfunction of an ion can be obtained from the wave function of the ground state of the molecule by acting with an electron annihilating operator:

$$
\left|\Psi_{n \sigma}^{+}\right\rangle=\mathbf{A}_{n \sigma}\left|\Psi_{0}\right\rangle \text {. }
$$

In the case of the SCF-based approach, leading to the Koopmans theorem, the operator $\mathbf{A}_{n \sigma}$ is the operator that annihilates an electron on one of the molecular orbitals. To represent the hole in the case of the correlated (APSLG) ground state, the concept of Dyson orbitals [23] can be used. These orbitals in a most general form are defined by the expression

$$
g_{n \sigma}(x)=\left\langle\Psi_{n \sigma}^{+}|\psi(x)| \Psi_{0}\right\rangle,
$$

where $\psi(x)$ is the fermion field operator that annihilates an electron in the point with the coordinates $x=(\mathbf{r}, \tau)$ (where $\tau$ stands for the spin projection of the annihilated electron). In the AO (or linear combination of $\mathrm{AO}$ ) representation, the Dyson orbitals are expressed as linear combinations of the respective basis functions. In the case of the SCF approach, Dyson orbitals coincide with molecular orbitals and, therefore, $\left|g_{n \sigma}\right\rangle=\mathbf{A}_{n \sigma}^{+}|0\rangle$. Within the APSLG-based scheme with the fixed geminals, the operators $\mathbf{A}_{n \sigma}$ are defined by the expression

$$
\mathbf{A}_{n \sigma}=\sum_{m}\left(\frac{\chi_{b_{m}}^{n}}{U_{m}} b_{m \sigma}+\frac{\chi_{a_{m}}^{n}}{V_{m}} a_{m \sigma}\right),
$$

whereas the Dyson orbitals are

$$
\left|g_{n \sigma}\right\rangle=\sum_{m}\left(\chi_{b_{m}}^{n} U_{m}\left|b_{m \sigma}\right\rangle+\chi_{a_{m}}^{n} V_{m}\left|a_{m \sigma}\right\rangle\right) .
$$

Therefore, one-electron functions $\mathbf{A}_{n \sigma}^{+}|0\rangle$ and $\left|g_{n \sigma}\right\rangle$ do not coincide. Moreover, these functions are not normalized. At the same time, we can check that $\left\langle 0\left|A_{n \sigma}\right| g_{m \tau}\right\rangle=\delta_{m n} \delta_{\sigma \tau}$, which serves as the biorthonormalization condition for the Dyson orbitals and the hole creation (electron annihilation) operators.

In the case of the APSLG approach with polarized geminals, the one-electron operators $\mathbf{A}_{n \sigma}$ cannot be determined easily. Therefore, to compare the localization of a hole for two APSLG-based methods, we use the concept of Dyson orbitals. In higher hydrocarbons, the Dyson orbitals obtained within both the computational schemes, which correspond to the lowest first ionization potential, are delocalized over the chain and are largely located on the $\mathrm{C}-\mathrm{C}$ bond backbone. This result coincides with that reported in Ref. [5]. In the case of the APSLGbased methods, the Dyson orbital is slightly more localized than in the case of the SCF method, but also has a sine-like form with the maximum at the center of the chain. The Dyson orbitals of the two APSLG-based methods are similar as well, but the charge distributions in the alkane cations in these two schemes differ noticeably. In the case of the APSLG-MINDO/3 method with the fixed geminals, the charges on the hydrogen atoms are very close to those in the neutral molecule, whereas in the case of the APSLG-MINDO/3 method with the polarized geminals, the charges on the hydrogen atoms became essentially - by 0.035 of unit charge (for hydrogens connected to the carbon atoms near the center of the chain) - more positive than in the neutral molecule, due to electron redistribution in the $\mathrm{C}-\mathrm{H}$ bonds, which are not affected directly by the ionization.

Let us consider ionization of molecules of other classes of compounds. In Table II, some results of the calculation on the first vertical ionization potentials by the APSLG-based method with fixed geminals are compared with the experimental data for some 


\section{TABLE II}

The first vertical ionization potentials $(\mathrm{eV})$ for some simple molecules obtained by the APSLG-based method with fixed geminals and the SCF-MINDO/3 method with use of the Koopmans theorem.

\begin{tabular}{lrrr}
\hline Molecule & APSLG & SCF & Exp. \\
\hline $\mathrm{H}_{2}$ & 15.614 & 15.636 & $15.43[24]$ \\
Cyclopropane & 10.058 & 10.357 & $11.0[25]$ \\
Cyclobutane & 10.130 & 10.434 & $10.6[26]$ \\
Cyclopentane & 10.697 & 11.075 & $10.50[27]$ \\
$\mathrm{C}_{2} \mathrm{H}_{4}$ & 10.418 & 10.388 & $10.51[28]$ \\
$\mathrm{NH}_{3}$ & 10.117 & 10.067 & $10.15[29]$ \\
$\mathrm{N}_{2} \mathrm{H}_{4}$ & 8.856 & 8.834 & $8.74[30]$ \\
$\mathrm{CH}_{3} \mathrm{NH}_{2}$ & 8.741 & 9.273 & $8.97[31]$ \\
$\mathrm{C}_{2} \mathrm{H}_{5} \mathrm{NH}_{2}$ & 8.789 & 9.246 & $8.66[31]$ \\
$\mathrm{H}_{2} \mathrm{O}$ & 12.794 & 12.765 & $12.62[20]$ \\
$\mathrm{CH}_{3} \mathrm{OH}$ & 10.781 & 11.089 & $10.84[32]$ \\
$\mathrm{C}_{2} \mathrm{H}_{5} \mathrm{OH}$ & 10.470 & 10.754 & $10.47[32]$ \\
\hline
\end{tabular}

simple molecules. Analysis of the data in Table II shows that the APSLG-MINDO/3-based method with fixed geminals gives values of the first vertical ionization potential that are close to experiment for all the molecules studied except cyclopropane. The divergence of calculated and experimental data obtained for cyclopropane almost vanishes in the case of higher cyclic hydrocarbons. Analysis of the wave function of the ionized state in the case of ammonia or water molecules showed that the main contribution to the hole is given by the electron lone pairs (73.1\% in the case of ammonia and $99.9 \%$ in the case of water).

The ionization potentials of methylamine and ethylamine are very close. This is due to the local character (lone pair) of the first vertical ionized state. This state is only slightly sensitive to the changes taking place far from the lone pair (the transition from the methylamine to ethylamine lowers the calculated first vertical ionization potential by less than $0.05 \mathrm{eV}$; the experimental change is also small). The changes in the local surrounding of the nitrogen atom such as the transition from ammonia to methylamine or from methylamine to dimethylamine stronger affects the first vertical ionization potential (by $1.4 \mathrm{eV}$ in the first case) in accordance with the experimental data. Notice that the alkyl substituents at the nitrogen atom increase the stability (lower the energy) of the first ionized state. This fact can be explained by taking into account the importance of the configuration with the ionized lone electron pair for this ionized state and the accepted (in organic chemistry) electron-donating character of alkyl groups (the matrix element of the Hamiltonian between the ionized states, where the remaining electron resides in the $\mathrm{HO}$ of the lone pair, and that with the remaining electron on the bonding orbital of the $\mathrm{N}-\mathrm{H}$ bond are $-3.92 \mathrm{eV}$ for ammonia and $-3.56 \mathrm{eV}$ for methylamine). The addition of alkyl groups to the oxygen atom of the water molecule decreases the first vertical ionization potential as well. The first vertical ionization potentials in the oxygen-containing molecules are sensitive as well to the kind and the number of alkyl substituents at the heteroatom (see Table II).

\section{Appendix}

The effective Hamiltonian for the $k$ th geminal in the field of other geminals and of an electron located on the bond spin-orbital $\left|c_{m \tau}\right\rangle$ of the $m$ th (ionized) bond $(k \neq m)$ can be presented as

$$
\begin{aligned}
H_{k c_{m \tau}}^{\text {eff }}=H_{k c_{m \tau}}^{\text {core }}+H_{k c_{m \tau}}^{1, \text { intra }}+ & H_{k c_{m \tau}}^{1, \text { inter }}+H_{k c_{m \tau}}^{\text {res }} \\
& +H_{k c_{m \tau}}^{2 \text {,intra }}+H_{k c_{m \tau}}^{2 \text {,inter }},
\end{aligned}
$$

where the contributions have the following meaning. The first term describes the attraction of electrons to the cores:

$$
H_{k c_{m \tau}}^{\text {core }}=\sum_{t \in\{r, l\}}\left(U_{k}^{t}-\sum_{B \neq T_{k}} \gamma_{T_{k} B} Z_{B}\right) \sum_{\sigma} t_{k \sigma}^{+} t_{k \sigma} .
$$

One-center repulsion of electrons of one bond gives the contribution to the Hamiltonian,

$$
H_{k c_{m \tau}}^{1, \text { intra }}=\sum_{t \in\{r, l\}}\left(t_{k} t_{k} \mid t_{k} t_{k}\right)^{T_{k}} t_{k \alpha}^{+} t_{k \beta}^{+} t_{k \beta} t_{k \alpha},
$$

and the one-center contribution from repulsion of the electrons of the $k$ th bond from the electrons of other bonds can be written as

$$
\begin{aligned}
& H_{k c_{m \tau}}^{1, \text { inter }}=\sum_{t \in\{r, l\}} \sum_{t_{q}^{\prime} \in T_{k}}\left[2\left(t_{k} t_{k} \mid t_{q}^{\prime} t_{q}^{\prime}\right)^{T_{k}}\right. \\
& \left.-\left(t_{k} t_{q}^{\prime} \mid t_{k} t_{q}^{\prime}\right)^{T_{k}}\right] P_{q}^{t^{\prime} t^{\prime}} \sum_{\sigma} t_{k \sigma}^{+} t_{k \sigma} \\
& +\sum_{t t^{\prime} \in\{r, l\}} \delta_{T_{k} T_{m}^{\prime}}\left(\eta_{m}^{t^{\prime} c}\right)^{2}\left[\left(t_{k} t_{k} \mid t_{m}^{\prime} t_{m}^{\prime}\right)^{T_{k}} \sum_{\sigma} t_{k \sigma}^{+} t_{k \sigma}\right. \\
& \left.-\left(t_{k} t_{m}^{\prime} \mid t_{k} t_{m}^{\prime}\right)^{T_{k}} t_{k \tau}^{+} t_{k \tau}\right] \text {. }
\end{aligned}
$$

The contribution from the intrabond resonance has the form

$$
H_{k c_{m \tau}}^{\mathrm{res}}=-\beta_{r_{k} l_{k}}^{R_{k} L_{k}} \sum_{\sigma}\left(r_{k \sigma}^{+} l_{k \sigma}+l_{k \sigma}^{+} r_{k \sigma}\right) .
$$


The contribution from the Coulomb repulsion between electrons located on the different atoms also can be divided into the intrabond,

$$
H_{k c_{m \tau}}^{2, \text { intra }}=\gamma_{R_{k} L_{k}} \sum_{\sigma} r_{k \sigma}^{+} l_{k-\sigma}^{+} l_{k-\sigma} r_{k \sigma},
$$

and the interbond

$$
\begin{aligned}
H_{k c_{m \tau}}^{2, \text { inter }}=\sum_{t \in\{r, l\}} \sum_{B \neq T_{k}} \gamma_{T_{k} B} \sum_{\substack{t_{q}^{\prime} \in B \\
q \neq k}}\left[\delta_{q m}\left(\eta_{m}^{t^{\prime} c}\right)^{2}\right. \\
\left.+2\left(1-\delta_{q m}\right) P_{q}^{t^{\prime} t^{\prime}}\right] \sum_{\sigma} t_{k \sigma}^{+} t_{k \sigma}
\end{aligned}
$$

contributions. The contributions of Eqs. (37) and (40) to the effective Hamiltonian are responsible for the polarization.

\section{ACKNOWLEDGMENTS}

This work was supported by the RFBR through grant 99-03-33176. One of us (A.M.T.) acknowledges financial support from Haldor Topsøe A/S. The authors thank the referee for valuable comments that helped to improve the manuscript.

\section{References}

1. Koopmans, T. Physica 1934, 1, 104.

2. Herman, M. F.; Freed, K. F.; Yeager, D. L. Adv Chem Phys $1981,48,1$

3. Cederbaum, L. S.; Domcke, W. Adv Chem Phys 1977, 36, 205

4. Hall, G. G. Proc Roy Soc Ser A 1951, 205, 541.

5. Pauzat, F.; Ridard, J.; Millié, Ph. Mol Phys 1972, 24, 1039.

6. Surjón, P. R. Topics in Current Chemistry; Springer-Verlag: Berlin, 1999; Vol. 203, p. 64.

7. Tokmachev, A. M.; Tchougréeff, A. L. Zh Fiz Khim 1999, 73, 320 [in Russian]; Russ J Phys Chem 1999, 73, 259 [English transl.].

8. Tokmachev, A. M.; Tchougréeff, A. L. J Comput Chem 2001, 22,752 .
9. Parks, J. M.; Parr, R. G. J Chem Phys 1958, 28, 335.

10. Surjón, P. R. Theoretical Models of Chemical Bonding; Springer-Verlag: Heidelberg, 1989; Part 2, p. 205.

11. Wu, W.; McWeeny, R. J Chem Phys 1994, 101, 4826.

12. Heitler, F.; London, F. Z Physik 1927, 44, 455.

13. Ovchinnikov, A. A. Z Struct Khim 1965, 6, 291 [in Russian].

14. Watanabe, K.; Nakayama, T.; Mottl, J. R. J Quantum Spectrosc Rad Transfer 1962, 2, 369.

15. Dewar, M. J. S.; Worley, S. D. J Chem Phys 1969, 50, 654.

16. Al-Joboury, M. L.; Turner, D. W. J Chem Soc 1964, 4434.

17. Nicholson, A. J. C. J Chem Phys 1965, 43, 1171.

18. Fueki, K. J Phys Chem 1964, 68, 2656.

19. Iskakov, L. I.; Potapov, V. K. Khim Vys Energii 1971, 5, 534 [in Russian].

20. Potts, A. W.; Price, W. C. Proc R Soc London Ser A 1972, 326, 165.

21. Traven, V. F. The Electronic Structure and Properties of Organic Compounds; Khimia: Moscow, 1989 [in Russian].

22. Cambi, R.; Ciullo, G.; Sgamellotti, A.; Tarantelli, F.; Fantoni, R.; Giardini-Guidoni, A.; Sergio, A. Chem Phys Lett 1981, 80, 295.

23. McWeeny, R. Methods of Molecular Quantum Mechanics, 2nd ed.; Academic Press: London, 1992.

24. Herzberg, G. J Mol Spectr 1970, 33, 147.

25. Turner, D. W.; Baker, C.; Baker, A. D.; Brundle, C. R. Molecular Photoelectron Spectroscopy; Wiley-Interscience: London, 1970.

26. Harrison, A. G.; Pottie, R. F.; Lossing, F. P. J Am Chem Soc 1961, 83, 3204

27. Pract, M. Th.; Derwiche, J. Chem Phys Lett 1970, 5, 546.

28. Brundle, C. R.; Robin, M. B.; Kuebler, N. A.; Basch, H. J Am Chem Soc 1972, 94, 1451.

29. Glushko, V. P., Ed. Thermal Constants of Substances, Handbook; VINITI: Moscow, 1965-1973; Vols. 1-7 [in Russian].

30. Franklin, J. L.; Dillard, J. G.; Rosenstock, H. M.; Herron, J. T.; Draxl, K. Ionization Potentials, Appearance Potentials, and Heats of Formation of Gaseous Positive Ions; National Bureau of Standards: Washington, DC, 1969.

31. Watanabe, K.; Mottl, J. R. J Chem Phys 1957, 26, 1773.

32. Refaye, K. M. A.; Chupka, W. A. J Chem Phys 1968, 48, 5205. 